\title{
Im Park übernachtet
}

\begin{abstract}
Nach einem Streit mit der Freundin wurde ein seit einem Tag vermisster 39-jähriger Mann vom Notarzt bei $-35^{\circ} \mathrm{C}$ auf einer Parkbank aufgefunden. Man bemerkte Alkoholgeruch in der Ausatmungsluft. Trotz dicker Winterkleidung und Winterstiefeln betrug die Körperkerntemperatur des Mannes nur noch $29^{\circ} \mathrm{C}$.
\end{abstract}

- Im Krankenhaus begann man mit Reanimationsmaßnahmen. Zusätzlich erhielt der Mann erwärmte Infusionslösungen und wurde von außen angewärmt. Die Reanimation war erfolgreich. An Armen, Beinen, Händen und Füßen bemerkte man aber eine ausgeprägte Blasenbildung.
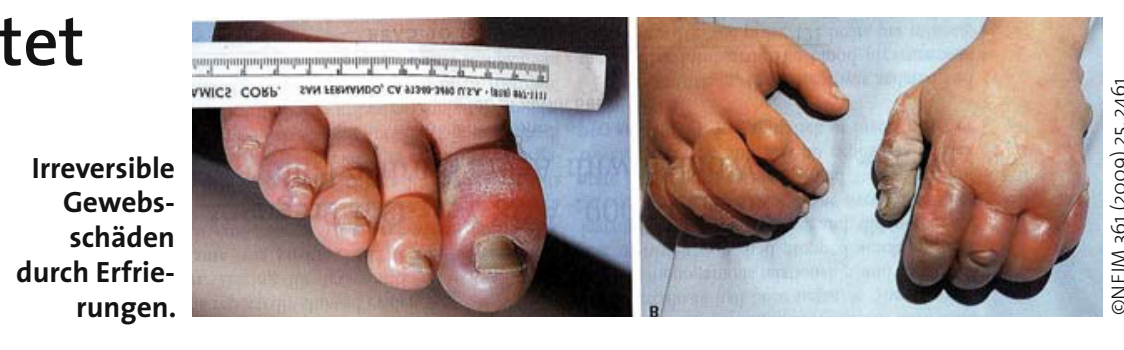

Die offensichtlichen Erfrierungen wurden täglich in einem warmen Bad behandelt und trocken verbunden, jedoch konnte man im Grunde nichts tun, als die Demarkierung der irreversiblen Gewebsschäden abzuwarten. Schließlich mussten die rechte Hand, der linke Mittelfinger und die linke Großzehe amputiert werden.

\section{Kommentar}

Das Risiko von Erfrierungen wird von vielen betroffenen Patienten nicht bedacht und falsch eingeschätzt. Insbesondere bei gleichzeitiger Alkoholintoxikation und durchnässten Schuhen kann es bereits bei Temperaturen nur um den Gefrierpunkt zu schweren Erfrierungen kommen. Der initiale Lokalbefund lässt häufig auch noch nicht erahnen, welche katastrophale Entwicklung sich nach wenigen Tagen hier anbahnt. H. S. FüEßL =

- H. Rehman, A. Seguin

(Regina Ou'Appelle Health Region Regina, SK, Canada. Frostbite. New Engl. J. Med. 361 (2009) 25, 2461

\section{H. pylori weg - Patient nimmt zu}

Die Eradikation des Helicobacter pylori gilt heute bei vielen Magenerkrankungen als Standard, insbesondere um ein Ulkusrezidiv bzw. ein Magenkarzinom zu verhindern. Allerdings nimmt nach einer erfolgreichen Ausrottung des Keims das Körpergewicht zu, und damit steigt auch das Risiko für eine erosive Refluxösophagitis an.

- Untersucht wurden 459 Patienten mit einem Helicobacter-pylori-positiven Ulcus duodeni ohne Refluxösophagitis. Zwei, sechs und zwölf Monate nach der Eradikation wurde der BMI bestimmt.

Nach einem Jahr hatte die Eradikation bei $20 \%$ der Patienten zu einem Anstieg des BMI um mehr als 1,5 kg/ $\mathrm{m}^{2}$ geführt. Bei 57 von 350 Patienten $(16,3 \%)$ entwickelte sich nach der Eradikation eine Refluxösophagitis. Es bestand eine enge Korrelation zwischen der Gewichtszunahme und dem Auftreten einer Refluxösophagitis.

\section{Kommentar}

Wo Licht ist, ist auch Schatten! Diese Untersuchung bestätigt frühere Beobachtungen, wonach eine erfolgreiche Eradikation des Helicobacter pylori zur Manifestation einer Refluxösophagitis führen kann. Bisher glaubte man, dass das $A b$ klingen der Gastritis zu einer vermehrten Säurebildung mit entsprechender Refluxsymptomatik führe. Die dauerhafte Abheilung des Ulkusleidens geht aber mit einer Gewichtszunahme einher, die wiederum einen Risikofaktor für die Refluxkrankheit darstellt. Ob dadurch aber auch das Risiko für ein Barrettkarzinom steigt, dafür gibt es bisher keine validen Daten. Somit dürften einige Ulkuspatienten „Magenpatienten" bleiben und evtl. wegen einer Refluxsymptomatik eine PPI-Therapie benötigen.

P. STIEFELHAGEN :

\section{- Y.-J. Yang et al.}

Increased body mass index after $\mathrm{H}$. pylor eradication for duodenal ulcer predisposes to erosive reflux esophagitis. J. Clin. Gastroenterol. 43 (2009) 705-710 